Recherches en didactique des langues et des cultures

Les cahiers de l'Acedle

$15-3 \mid 2018$

Quelles médiations en didactique des langues et des cultures?

\title{
Médiation, discursivité et compétence pragmatique
}

Des textes de la presse écrite quotidienne aux pratiques d'enseignement du FLE

Florimond Rakotonoelina

\section{OpenEdition}

\section{Journals}

Édition électronique

URL : http://journals.openedition.org/rdlc/3524

DOI : $10.4000 /$ rdlc.3524

ISSN : 1958-5772

Éditeur

ACEDLE

Référence électronique

Florimond Rakotonoelina, " Médiation, discursivité et compétence pragmatique », Recherches en didactique des langues et des cultures [En ligne], 15-3 | 2018, mis en ligne le 01 septembre 2018 , consulté le 03 mai 2019. URL : http://journals.openedition.org/rdlc/3524 ; DOI : 10.4000/rdlc.3524

Ce document a été généré automatiquement le 3 mai 2019.

\section{$\Theta \Theta \Theta \Theta$}

Recherches en didactique des langues et des cultures is licensed under a Creative Commons AttributionNonCommercial-NoDerivatives 4.0 International License 


\title{
Médiation, discursivité et compétence pragmatique
}

Des textes de la presse écrite quotidienne aux pratiques d'enseignement du FLE

\author{
Florimond Rakotonoelina
}

1 Le recours aux textes de la presse écrite quotidienne dans les pratiques d'enseignement du français, langue étrangère, a toujours joué un rôle fondamental depuis l'avènement des approches communicatives ${ }^{1}$ et il en va de même dans la perspective actionnelle depuis les années $2000^{2}$. Ce recours se manifeste matériellement par l'insertion d'articles dans les manuels de langue ou par la distribution de photocopies en classe, mais cette extraction des textes de leur support d'origine a conduit ces textes dits d'information médiatique à perdre leur "logique pragmatique», pour reprendre les termes de Charaudeau (2010). En effet, les textes de la presse écrite quotidienne font état de l'actualité correspondant aux informations du moment mais, une fois insérés dans les manuels, voire photocopiés et distribués, ils perdent ce pouvoir de représenter l'actualité pour servir d'autres fins, le plus souvent d'ordre socioculturel ${ }^{3}$. D'un point de vue didactique, la question est la suivante : peut-on enseigner l'actualité en classe de langue? Autrement dit, peut-on traiter les textes de la presse quotidienne pour ce qu'ils sont au premier abord, c'est-à-dire le reflet de l'actualité ? Dans l'affirmative, la question est dès lors de savoir comment enseigner cette actualité. Pour y parvenir, on propose ici de l'aborder à partir de la notion d'événement médiatique, ce qui permettra d'envisager les textes, non plus comme des entités indépendantes les unes des autres, mais comme des instances de discours représentant une actualité itérative ${ }^{4}$. Dans ces circonstances, l'enseignant doit jouer un double rôle : celui de médiateur des textes de la presse écrite quotidienne en classe de langue d'une part, ce qui implique qu'il opère différents types de choix; celui de médiateur des savoirs langagiers sur ces textes d'autre part, ce qui suppose le développement d'une compétence pragmatique chez les apprenants permettant de mettre au jour des mises en scène discursives en fonction des événements que représente l'actualités. Par-delà ce double rôle, il s'agit pour l'enseignant de français, langue étrangère, de comprendre et de faire comprendre à des apprenants comment 
l'information est médiatisée par la presse écrite d'une langue-culture ${ }^{6}$ à partir des préconisations, on le verra, du CECRL ${ }^{7}$.

2 On distinguera deux types de médiation, une médiation communicationnelle pour comprendre les phénomènes de l'actualité médiatique et une médiation didactique liée au travail de l'enseignant chargé de rendre accessible cette actualité auprès des apprenants. L'objectif de cet article sera d'ordre méthodologique d'une part, ce qui permettra de poser un cadre de réflexion, et d'ordre descriptif d'autre part, montrant en quoi l'analyse des textes participe à la compréhension de l'actualité.

\section{De la médiation communicationnelle à la médiation didactique}

3 On envisagera la médiation de deux points de vue : la médiation comme impliquant une séparation et la médiation comme supposant une réunion.

\section{Ce qui sépare les apprenants de l'actualité}

4 Debon, s'appuyant sur Jacquinot-Delaunay (2003), rappelle que « [1]a médiation, c'est ce qui relie, ce qui sert d'intermédiaire entre des situations et des acteurs, sans oublier que la séparation est présente en amont et en aval » (Charlier, Debon, Hülsmann et Tait, 2013 : \$15). Plaçant notre travail à l'interface de l'analyse du discours et de la didactique des langues étrangères (voir Rakotonoelina, 2007a et 2007b; Peytard et Moirand, 1992), la notion de séparation, centrale dans cette définition, suppose que les journalistes, à travers la presse écrite quotidienne, se servent des textes pour re-présenter l'actualité, ce qui signifie qu'il n'y a pas de correspondance biunivoque entre l'actualité et les textes qui la représentent ${ }^{8}$. Dans ces circonstances, l'enseignant de langue étrangère, en tant qu'intermédiaire, doit permettre à l'apprenant d'accéder à ces modes de représentation ethno-centrés de l'actualité.

5 Mais cette séparation s'opère également au sein des textes eux-mêmes: l'analyse du discours postule que les textes sont polysémiques et qu'il n'y a pas de correspondance biunivoque entre les formes langagières et leur interprétation'. Si le discours est ainsi « divisé » et s'il génère de multiples interprétations, c'est qu'il y a, de part et d'autre des messages, "deux idiolectes », celui de l'énonciateur et celui du co-énonciateur, qui font que les messages ne passent pas dans leur totalité, comme le souligne Kerbrat-Orecchioni (1980: 16) dans sa longue critique du schéma de la communication de Jakobson. L'enseignant de langue étrangère aura alors également pour tâche, en tant qu'intermédiaire, de guider l'apprenant dans l'interprétation des textes représentant l'actualité à partir de «la matérialité » des formes linguistiques; il s'agit donc, pour l'apprenant, d'émettre des hypothèses sur le « vouloir dire » des textes en prenant appui sur les marques formelles.

6 Enfin, on distinguera ici une dernière séparation entre les textes et leurs récepteurs; les messages véhiculés par les textes n'ont d'existence que par l'intermédiaire d'une interaction entre les textes et les récepteurs qui leur donnent une cohérence (Charolles, 1995). En effet,

un texte est un message virtuel, une communication potentielle : il est le reflet d'un acte de discours (dont le scripteur est l'initiateur), mais ne devient communication 
que grâce à un lecteur, qui, apportant à ce processus interactif qu'est la compréhension une multiplicité de connaissances et de savoir-faire, interprète les signaux contenus dans le texte et se construit une représentation - nécessairement idiosyncratique - de ce que le scripteur a pu vouloir dire (Péry-Woodley, 1993 : 59-60).

7 L'enseignant de langue étrangère, en tant qu'intermédiaire, ne participe plus seulement à la transmission de savoirs langagiers; il montre comment l'agencement de ces savoirs dans la presse quotidienne constitue des indices permettant la construction d'une représentation partagée de l'actualité, laquelle, comme on l'a vu, doit être interprétée en lien avec son moment et son lieu d'énonciation.

On propose de récapituler ces trois séparations au moyen du schéma suivant :

\section{Schéma 1 - Médiation et séparations « communicationnelles »}

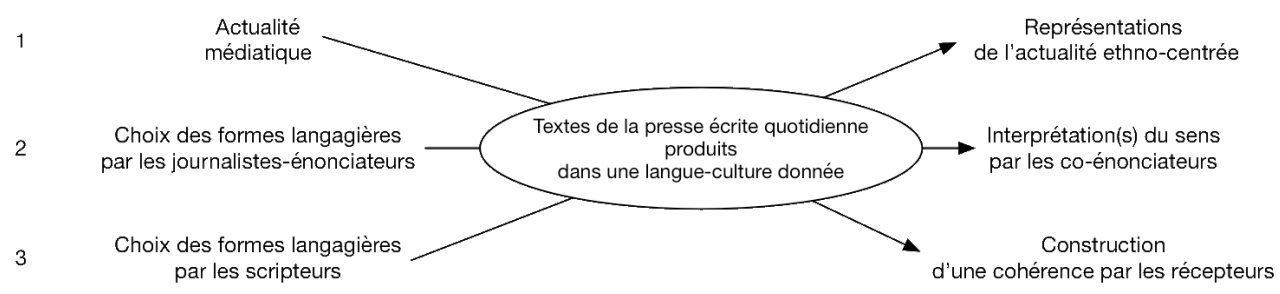

9 En définitive, c'est cette triple brèche liée à la production et à l'interprétation des textes dans des cadres spatio-temporels déterminés que l'enseignant de la langue étrangère viendrait combler. En d'autres termes, l'apprenant devrait être familiarisé avec un type de médiation communicationnelle liée à l'actualité médiatique ${ }^{10}$.

\section{Comment réconcilier la classe de langue avec l'actualité ?}

Rézeau rappelle ce que serait la médiation dans le domaine de la didactique :

- ensemble des aides - personnes et instruments - mises à la disposition de l'apprenant pour faciliter provisoirement son appropriation de la L2 et l'utilisation autonome ultérieure de celle-ci ;

- action de la personne qui facilite l'apprentissage de la L2 par une relation d'aide, de guidage, avec ou sans instruments (2002: §36).

11 Cette double définition, positive, met l'accent sur les actions permises par l'étendue des moyens en général («instruments») et par les actants («personne(s)») qui rendent possibles la réunion d'éléments scindés. À ce titre, il s'agit maintenant de proposer des moyens par lesquels l'enseignant de langue assurerait la jonction des trois séparations qui viennent d'être explicitées.

12 Si l'on part du principe que les textes de la presse quotidienne doivent être traités en classe de langue en lien avec ce qu'ils représentent, à savoir l'actualité, c'est parce que c'est ainsi que les locuteurs d'une langue les perçoivent prioritairement quand ils en font lecture. De ce point de vue, il n'y a pas lieu de priver les apprenants d'une langue étrangère de «cette réalité ». Néanmoins, cette compétence de compréhension de l'actualité ne signifie pas pour autant qu'elle serait accessible à tous les niveaux d'apprenants. En effet, s'agissant de compréhension écrite, et suivant en cela les descripteurs du CECRL, le niveau minimum attendu pour un travail de compréhension de l'actualité se situerait au niveau B2 ${ }^{11}$. Par ailleurs, travailler sur l'actualité suppose 
d'intégrer en classe de langue des textes produits sinon le jour même, au moins dans la semaine, ce qui peut représenter une difficulté. C'est la raison pour laquelle on propose de saisir l'actualité à partir de la notion d'événement médiatique d'une part, et de ne plus considérer le texte d'actualité comme une entité isolée mais pris dans un réseau multiple d'autres textes représentant cette même actualité et déterminés par des caractéristiques formelles identiques d'autre part.

La notion d'événement médiatique permet de saisir l'actualité comme elle se présente et de rattacher toute actualité à un événement. On définit l'événement médiatique comme un fait marquant pour les médias, c'est-à-dire un fait d'actualité relayé par plusieurs titres de presse qui peut être de courte durée (une seule journée, comme c'est le cas de la journée annuelle de l'asthme) ou d'une durée plus longue (de plusieurs jours à plusieurs semaines, voire plusieurs mois, comme cela peut être le cas d'une campagne électorale). Par ailleurs, un événement médiatique est un événement cyclique : par exemple, les annonces de grève, les catastrophes naturelles, les attentats terroristes, les hommages aux victimes, les manifestations, etc. font régulièrement l'objet d'articles de presse. Enfin, un événement médiatique propose toujours d'observer l'actualité sous un certain angle d'attaque (voir infra). Ainsi, en rapportant l'actualité à l'événement type qu'elle représente, il devient aisé de systématiser l'usage des textes de la presse écrite dans les pratiques d'enseignement. Croyant travailler sur l'actualité « récente » à partir de textes récents, l'apprenant travaille en fait sur l'événement que représente cette actualité puisque cet événement n'est que la répétition d'actualités passées ou d'actualités à venir. L'enseignant recourt donc à différents types d'événements médiatiques en classe - en s'appuyant sur l'actualité du moment - pour préparer les apprenants à des tâches authentiques de lecture de la presse quotidienne.

Pour accéder à cette représentation ethno-centrée de l'événement médiatique lié à l'actualité, l'enseignant de langue peut s'appuyer, selon nous, sur trois concepts opératoires, qui figurent tous derrière ce que recouvre l'acquisition d'une compétence pragmatique en langue étrangère dans le CERCL. Autrement dit, comprendre l'actualité, c'est « faire jouer » cette compétence. Ainsi, la compétence pragmatique est définie de la manière suivante :

La compétence pragmatique recouvre l'utilisation fonctionnelle des ressources de la langue (réalisation de fonctions langagières, d'actes de parole) en s'appuyant sur des scénarios ou des scripts d'échanges interactionnels. Elle renvoie également à la maitrise du discours, à sa cohésion et à sa cohérence, au repérage des types et genres textuels [...]. Plus encore pour cette composante que pour la composante linguistique, il n'est guère besoin d'insister sur les incidences fortes [...] des environnements culturels dans lesquels s'inscrit la construction de telles capacités $(2001: 18)^{12}$.

Le premier concept opératoire, permettant de décrire et de réunifier les premiers éléments disjoints, correspond à ce qu'on appelle l'angle d'attaque puisqu'il détermine l'ensemble des faisceaux descriptifs sélectionnés et partagés par les journalistes pour " traduire » une actualité afin d'en donner une vision ethno-centrée ; on proposera une transposition du «type textuel descriptif» (Adam, 1992) pour en rendre compte (voir infra 2.1. $)^{13}$.

La compétence pragmatique se subdivise en deux compétences: la compétence fonctionnelle et la compétence discursive (id. : 96-98). La compétence fonctionnelle recouvre l'utilisation, entre autres, "des textes écrits en termes de communication à des fins fonctionnelles particulieres" (id.: 98). C'est dans cette 
compétence que l'on puisera le deuxième concept opératoire qui servira à la description de l'actualité, à savoir le concept d'acte illocutoire d'Austin (1962), plus connu dans les approches notionnelles-fonctionnelles sous l'appellation d'acte de parole. L'apprenant ne pourrait ainsi accéder à l'interprétation/compréhension d'un événement représentant une actualité que dans la mesure où il aurait la capacité de déterminer l'ensemble des actes sous-jacents à cet événement. Il convient donc pour l'enseignant de langue de permettre à l'apprenant d'opérer un chemin inverse, qui va des énoncés aux actes et non plus des actes aux énoncés. Ce faisant, il s'agit de comprendre le "vouloir dire» de l'actualité sous forme d'actes de parole, et ainsi répondre à la question de la deuxième séparation (voir infra 3.1.).

18 La compétence discursive, pour sa part, traite de la connaissance que l'utilisateur/ apprenant a des principes selon lesquels les messages sont organisés, structurés et adaptés (id. : 96). Ainsi le troisième concept, qui peut apporter une solution à la troisième séparation, est celui de " cohérence », cohérence que l'on peut chercher à reconstruire à partir d'une analyse de la structuration thématique (id.) et de la cohésion (id.) ${ }^{14}$. Il s'agit donc pour l'enseignant de langue d'aider l'apprenant à comprendre l'organisation thématique d'une actualité autant que les éléments qui participent à sa cohésion (voir infra 3.2.).

En définitive, le recours à ces trois principaux concepts (angle d'attaque, acte et cohérence) sera un moyen pour l'enseignant de faire comprendre l'actualité par l'apprenant ; mais, au-delà de cette compréhension, il s'agit de lui permettre de dégager une schématisation discursive de l'événement médiatique sous-jacent à l'actualité pour faciliter sa lecture de la presse écrite en dehors de la classe. Plus précisément, il s'agit de «théoriser ", à partir d'une actualité particulière, l'événement type que cette dernière représente et de dégager trois types de schématisations discursives : une schématisation fonctionnelle visant à reconstruire les actes, une schématisation thématique et une schématisation liée aux phénomènes de cohésion portant sur la construction d'une cohérence. Dès lors, la "schématisation apparaît ainsi non tellement comme l'expression discursive d'un sens que comme une sollicitation à construire un sens » (Grize, 1996:118) ${ }^{15}$.

On peut ainsi récapituler cette démarche sous la forme du schéma suivant :

Schéma 2 - Jonctions et médiation didactique
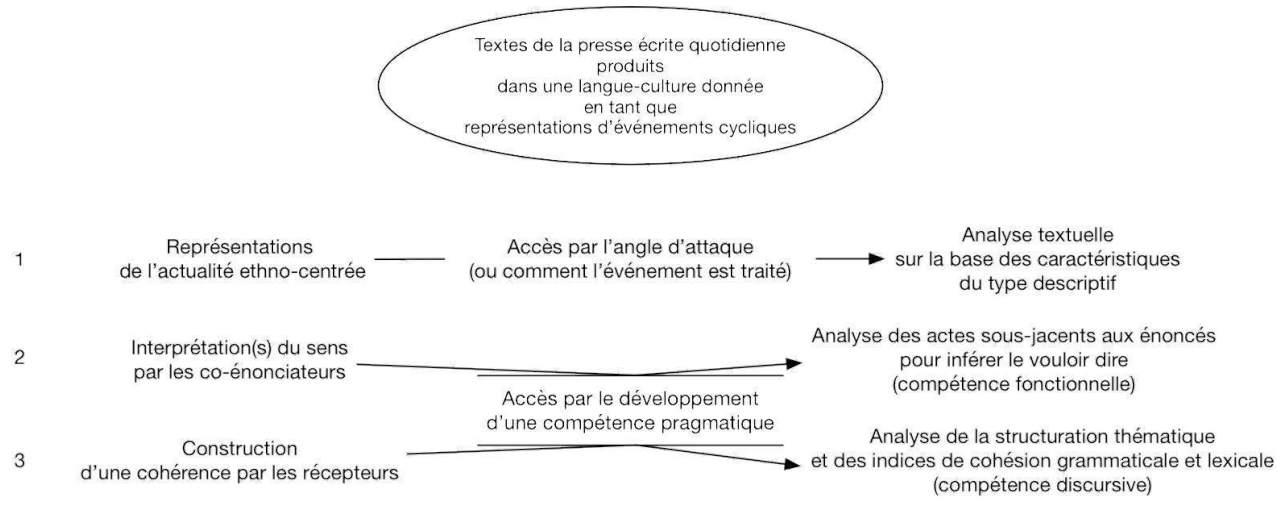

Il s'agira, dans la suite du propos, d'illustrer la manière dont la presse quotidienne construit des angles d'attaque autour d'événements donnés, comment un corpus peut permettre de les analyser et comment s'opère la récursivité des événements. Puis, on montrera comment s'élaborent les schématisations discursives. 


\section{Événement médiatique : angle d'attaque de l'événement et sa récursivité}

21 Un angle d'attaque peut être considéré comme la manière dont la presse nationale conçoit l'actualité liée à un événement. Pour appréhender cette médiation, on recourt au "type descriptif» (Adam, 1992) pour montrer qu'un événement médiatique, dans une culture donnée, n'est ni plus ni moins qu'une projection typique de la manière dont les journalistes se représentent l'événement. Dans les pratiques d'enseignement, il s'agit de faire comprendre comment la presse écrite construit cet événement dans la langue cible dans un espace géographique déterminé.

\section{Saisir l'actualité par l'intermédiaire de son angle d'attaque} propres propriétés, ses propres aspects, ses propres mises en relation, etc. Par exemple, 
dans la version web du Monde daté du 14 janvier 2017, un article s'intitule « Neige : 23000 foyers toujours privés d'électricité »; par une procédure d'enchâssement, qui se trouve être ici une conséquence du froid, le dernier paragraphe de l'article glisse sur les dispositions prises par la mairie de Paris :

La mairie de Paris a quant à elle annoncé renforcer dès ce week-end son offre d'hébergement pour les sans-abri avec l'ouverture supplémentaire d'une salle dans le $4 \mathrm{e}$ arrondissement et d'un gymnase dans le $15^{\mathrm{e}}$, soit 85 places.

Cet enchâssement n'était pas nécessairement attendu : pourquoi la mairie de Paris et non celle de Lyon? pourquoi indiquer le lieu et le nombre d'offres d'hébergement dans un article dont le thème-titre était les coupures d'électricité ?

Ces procédures d'enchâssement jouent un rôle particulier dans la médiation d'une actualité dans la mesure où elles participent, au même titre que les procédures d'aspectualisation et/ou d'assimilation, à une mise en scène discursive culturellement située de l'événement. Pour comprendre un événement dans une langue-culture donnée, ce sont ces procédures d'enchâssement que les apprenants d'une langue étrangère devront identifier au même titre que les procédures d'aspectualisation.

Comment construit-on un corpus autour d'un événement médiatique ? Un tel corpus doit servir de référence à l'enseignant de langue pour montrer non seulement comment un événement singulier fonctionne, mais aussi comment, par un travail centré sur l'acquisition d'une compétence pragmatique autour de la presse quotidienne, l'événement singulier rejoint la catégorie des événements cycliques.

\section{Saisir l'événement par l'intermédiaire d'un corpus (unilingue ou plurilingue?)}

Un corpus autour d'un événement médiatique suppose de récupérer non plus un article, mais plusieurs articles issus de titres de presse différents, l'objectif n'étant plus de travailler sur un article de presse, comme moteur de compréhension écrite et de discussion souvent culturelle, mais sur plusieurs articles simultanément pour que l'apprenant puisse recentrer son attention sur les problématiques liées à la mise en scène de l'actualité et sur la discursivité des événements dans la presse.

31 Par exemple, un corpus qui donne lieu à un événement type « Résultats d'une enquête de police suite à un braquage sur une personne connue » peut être issu d'un événement particulier, en l'occurrence celui du vol de bijoux de Kim Kardashian en France. Un vol de bijoux ne reflète pas, en lui-même, un événement médiatique. L'événement médiatique implique nécessairement un point de vue particulier culturellement situé, ce qui entraîne des procédures d'aspectualisation qui peuvent porter sur l'enquête policière en cours suite à ce braquage. Ainsi, si l'on observe le traitement médiatique de cet événement en France, on constate, du moins pour la seule date du 10 janvier 2017, que c'est par le biais de procédures d'assimilation que la presse a choisi de présenter les résultats de l'enquête. Cette assimilation porte sur le parallélisme que l'on peut faire entre cette enquête et le milieu du grand banditisme français des années 1960 jusqu'à la fin des années 1980 (par exemple, en référence au gang des Lyonnais) ou sur la mise en relation avec des films célèbres français qui en traitent comme Les tontons flingueurs. Cela se reflète dans les titres des articles. Par exemple, le Parisien propose le titre suivant: "Braquage de Kim Kardashian: une affaire de "truands à l'ancienne" ». L'Express, pour sa part, propose: 
"Braquage de Kim Kardashian: des "papys gangsters" au cour de l'action ». Enfin, du côté du nouvel Obs, on lit: "Affaire Kim Kardashian: Ocean's Twelve à la française ». Même si cette procédure d'assimilation n'est pas comprise des apprenants de la langue étrangère, il n'en demeure pas moins que les configurations discursives des articles eux-mêmes sont tout à fait différentes des configurations proposées par la presse étrangère en raison justement de ces procédures. Celles-là diffèrent par exemple dans le cas de la presse américaine qui, à la même période, se centre essentiellement sur le chauffeur. Le 9 janvier, le US Daily News propose comme titre : «Kim Kardashian's limo driver in Paris among 17 suspects arrested for burglary»; le lendemain, le 10 janvier, le Los Angeles Times a quasiment un titre similaire, laissant supposer un retour sur le chauffeur : «Kim Kardashian's Paris chauffeur among 17 robbery suspects arrested in France ». Les deux articles américains abordent de façon similaire et de manière lapidaire l'identité des suspects, ce qui est loin d'être le cas des journaux français.

\section{Saisir l'événement typique par l'intermédiaire de l'événement spécifique}

L'événement spécifique que l'on vient de présenter est pris dans la spirale d'autres événements du même type qui font partie du paysage médiatique de la France, d'où la désignation de l'événement sous une forme abstraite "Résultats de l'enquête de police suite à un braquage impliquant une personne connue». Ainsi, on constate que les braquages, qui relèvent généralement des faits divers, occupent une place médiatique importante lorsque le braquage implique soit une somme importante (bijouterie, fourgon) soit une personne connue. À partir d'un événement similaire à venir, il sera facile non seulement de traiter de l'actualité dans les pratiques d'enseignement, mais également d'aborder l'événement du point de vue de sa projection typique. Pour faire le parallèle avec des événements du même type, passés cette fois, on peut citer le braquage d'un prince saoudien à Paris en 2014 ; le Point intitulait son article "Audacieux braquage d'un prince saoudien en plein Paris » (18 avril 2014). Et quelques mois après, à l'instar du braquage de Kardashian, la presse faisait état des résultats de l'enquête de police; par exemple, le Monde titrait en mai 2015 "Six suspects mis en examen et incarcérés pour le braquage d'un convoi saoudien à Paris » (23 mai 2015).

Dans ces circonstances, l'enseignant de la langue étrangère devient le médiateur entre l'actualité et l'événement que cette actualité représente en traitant l'actualité comme autant d'occurrences d'événements typiques. À partir l'analyse de cet événement, il s'agit maintenant d'observer comment l'apprenant acquiert une compétence pragmatique liée aux textes de la presse écrite quotidienne.

\section{Acquisition d'une compétence pragmatique liée à l'actualité}

Pour acquérir une compétence pragmatique liée à la compréhension de la presse écrite quotidienne, l'apprenant doit être capable d'identifier les intentions sous-jacentes aux énoncés qui constituent le message représentant l'événement (les actes de parole) et d'analyser les formes langagières qui participent à la construction d'une cohérence (structuration thématique et phénomènes de cohésion). On illustrera ici l'analyse des 
actes (compétence fonctionnelle) et l'analyse des indices participant de la cohérence (compétence discursive) en limitant ces derniers aux phénomènes de cohésion lexicale ${ }^{16}$.

\section{L'interprétation des actes sous-jacents à l'événement}

À partir des énoncés des différents articles du corpus relatifs aux "Résultats d'une enquête de police suite à un braquage sur une personne connue ", il s'agit de reconstruire les visées illocutoires de l'événement, susceptibles de se réactualiser si un événement du même type devait se reproduire. L'analyse comparative des énoncés des textes permet de dégager les actes suivants :

- Faire état de l'avancée de l'enquête

- Expliquer le travail des enquêteurs

- Faire état de ce que pensent les enquêteurs

- Rappeler le déroulement du braquage

- Donner la liste des malfaiteurs

- Rendre compte des malfaiteurs

- Pointer le cerveau de l'opération

- Présenter les malfaiteurs individuellement ou collectivement

- Donner l'origine des malfaiteurs

- Donner la moyenne d'âge des malfaiteurs

- Faire état des indices ayant permis de remonter les pistes et à l'origine des arrestations

- Livrer les chefs d'accusation et l'état des gardes à vue

- Donner des nouvelles du statut du butin

L'obtention de ces fonctions résulte de l'interprétation des indices formels présents dans les textes. Par exemple, pour la fonction « rappeler le déroulement du braquage », c'est à partir de l'énoncé suivant qu'il est possible d'inférer le «vouloir dire» de ces indices (en italiques) :

La vedette ultramédiatisée de 36 ans a été braquée par cinq hommes armés dans une discrète résidence hôtelière de luxe du VIIIe arrondissement de Paris. Après l'avoir ligotée, bâillonnée et enfermée dans la salle de bain, ils étaient repartis avec les bijoux.

\section{L'interprétation des marques lexicales}

L'interprétation des marques lexicales s'opère à partir d'une analyse de la cohésion lexicale. Il s'agit, pour chaque texte, de mener ce travail pour dégager ensuite des invariants. On entend par cohésion lexicale deux types de relation que contractent entre eux les mots d'un texte :

- il peut s'agir de relations lexicales (relations de ressemblance, de hiérarchie et de solidarité) ; ce sont des relations inscrites en langue dont on observe le fonctionnement en discours ;

- il peut s'agir de phénomènes de co-référence parallèle, où la mise en relation entre les mots n'est plus inscrite en langue, mais s'opère en discours. régularités observables au niveau des types de relation, mais c'est aussi comprendre le 
stock lexical qui est en jeu lors de la mise en discours/médiation d'un tel événement. De même, c'est être capable de dire quels termes co-réfèrent pour un événement donné.

Les relations de solidarité (partie/tout), pour cet événement, sont les moins nombreuses : on relève des relations entre gang/malfaiteur (un gang étant une bande de malfaiteurs), mais également entre enquête/perquisition (l'enquête étant un ensemble de recherches dont fait partie la perquisition) et entre équipe/personne (une équipe étant constituée de personnes). Du côté des relations de ressemblance, on constate que certaines sont symptomatiques des procédures d'assimilation qu'on a décrites plus haut; ainsi, on rapproche cerveau/chef, enquête/investigations (au pluriel), équipe/équipage, garde du corps/bodygard, policier/flic. Des termes comme «cerveau» ou "flic» projettent l'événement dans l'histoire du grand banditisme français signalée plus haut. C'est du côté des relations de hiérarchie que l'on observe un nombre important d'unités reliées à l'hyperonyme "personne». Surtout, on constate que ces relations sont centrées quasiment uniquement sur un seul référent, le ou les malfaiteur(s). On relève ainsi, à partir du lexème hyperonymique "personne ", «suspect ", "voyou », " doyen", " septuagénaire », « malfaiteur ", « homme », « chauffeur ", " gardé à vue », « braqueur ", " truand », « caïd », « interpellé », « agresseur », « intermédiaire », « costaud » (nominalisé), «type », « auteur », "chef », etc. On pourrait naturellement dégager des relations plus fines entre ces unités, par exemple entre «caïd» et "chef» ou entre «truand» et «malfaiteur»; cependant, l'objectif est bien ici d'identifier, à partir de "personne », comment s'opèrent les différentes nominations en discours des interpellés.

Enfin, contrairement aux relations de hiérarchie qui portent sur un même référent (singulier ou pluriel), les phénomènes de co-référence parallèle visent des référents distincts : d'une part, les protagonistes de l'histoire; d'autre part, des lieux et objets de l'affaire. On ne donnera ici que quelques exemples saillants. Ainsi, la principale protagoniste, Kim Kardashian reçoit son lot de désignations: "la star américaine », la «star ", «la starlette», «la victime », «la vedette ultramédiatisée », «la cible » et «l'ultraplantureuse Kim ». Du côté des malfaiteurs, on rencontre « le doyen/un dénommé Pierre ", «Pierrot/le malfrat ", "les malfaiteurs/les papys gangsters", "son frère/le jeune employé », « le chauffeur/cet employé », « un jeunot/un habitant du Val-de-Marne de 60 ans ». Du côté des autres personnages, on aura « le garde du corps/une baraque » et « les enquêteurs/les policiers ». Enfin, en ce qui concerne les lieux et les objets, on aura par exemple "hôtel particulier/immeuble ", «butin » pour référer à "bijoux » et « argent ».

41 À travers ces quelques exemples d'analyse lexicale, on voit comment se dessine la médiation de cet événement à travers la presse, mais on voit aussi l'intérêt pour l'apprenant d'appréhender lexicalement l'événement pour mieux le situer linguistiquement et culturellement.

\section{Conclusion}

En tant qu'analyste du discours, notre travail consiste à articuler les recherches sur les discours avec la didactique des langues étrangères pour faire évoluer les pratiques d'enseignement, notamment celles liées à la compétence pragmatique, cette dernière se centrant sur les configurations linguistiques et discursives des textes et des discours. La discursivité liée à l'actualité en langue cible n'a rien d'évident pour des apprenants d'une langue étrangère ; lire des nouvelles, notamment à partir du niveau B2, ne doit pas être 
un parcours du combattant sous prétexte que l'apprenant n'est pas aguerri à la mise en scène de l'actualité dans la langue cible. À partir du concept de médiation, on a décrit la médiation communicationnelle de manière opératoire dans le contexte des pratiques d'enseignement à partir de la notion, plus abstraite, de séparation : entre l'actualité et sa représentation, l'angle d'attaque joue un rôle de médiation ; la médiation entre les formes langagières et leur sens en discours est assurée par des hypothèses sur leur "vouloir dire "; enfin, si les textes sont reliés à un sens, c'est parce que le lecteur s'accorde à leur conférer une cohérence. La médiation didactique vise à faciliter l'appropriation de cette médiation communicationnelle. Pour cela, l'enseignant doit constituer des corpus qui permettent de ramener l'actualité à des événements types, lesquels se distinguent par des schématisations discursives spécifiques: il aide ainsi à la mise au jour de l'angle d'attaque ; il fait comprendre le sens de l'information à partir de la visée illocutoire des énoncés ; il participe à la construction d'une interprétation cohérente des textes. À l'issue de ce travail sur les événements médiatiques, il est possible de dresser une cartographie de leur schématisation en fonction de l'actualité, des envies ou des besoins des apprenants, ce qui peut les inciter à se perfectionner en compréhension écrite de la presse quotidienne par le truchement des événements préalablement étudiés. Mais l'enseignant de langue peut également y trouver son compte: travailler sur les événements que représente l'actualité évite de s'installer dans une routine puisque l'actualité est chaque fois différente tandis que les événements, eux, sont cycliques ; ainsi, son travail se trouve allégé par l'articulation de la nouveauté (l'actualité) et du connu (les schématisations élaborées antérieurement).

\section{BIBLIOGRAPHIE}

Adam, J.-M. (1992). Les textes : types et prototypes. Récit, description, argumentation, explication et dialogue. Paris : Nathan.

Austin, J. L. (1962, 1970). Quand dire, c'est faire. Paris : Seuil.

Benveniste, É. (1974). Problèmes de linguistique générale 2, Paris : Gallimard.

Conseil de l'Europe (2001). Cadre européen commun de référence pour les langues. Paris : Conseil de l'Europe/Didier.

Charaudeau, P. (2010). «Une éthique du discours médiatique est-elle possible ? ». Communication, vol. 27, $\mathrm{n}^{\circ}$ 2. pp. 51-75. http://communication.revues.org/3066.

Charlier, B., Debon, C., Hülsmann, T. \& Tait, A. (2013). « Médiation, médiations... ». Distances et

médiations des savoirs, $\mathrm{n}^{\circ}$ 3. http://journals.openedition.org/dms/276.

Charolles, M. (1995). «Cohésion, cohérence et pertinence du discours ». Travaux de linguistique, $\mathrm{n}^{\circ}$ 29. pp. 125-151.

Grize, J.-B. (1996). Logique naturelle et communications. Paris : PUF.

Jacquinot-Delaunay, G. (2003). « Médiation, médiatisation, un entre-deux ». Notions en question, $\mathrm{n}^{\circ}$ 7. pp. 127-136. 
Halliday, M. A. K. \& Hasan, R. (1976). Cohesion in English. Londres: Longman.

Kerbrat-Orecchioni, C. (1980). L'énonciation. De la subjectivité dans le langage. Paris : Armand Colin.

Le Goffic, P. \& Besse, H. (1970). « Le contenu linguistique du N2 », Le français dans le monde, n $^{\circ} 73$. pp. 15-21.

McCarthy, M. (1991). Discourse Analysis for Language Teachers. New York: Cambridge University Press.

Münchow (von), P. (2004). Les journaux télévisés en France et en Allemagne. Plaisir de voir ou désir de s'informer. Paris : Presses Sorbonne Nouvelle.

Péry-Woodley, M.-P. (1993). Les écrits dans l'apprentissage. Paris : Hachette.

Peytard, J. \& Moirand, S. (1992). Discours et enseignement du français. Les lieux d'une rencontre. Paris : Hachette.

Rakotonoelina, F. (2017a). « Pour une éthique des textes et des discours d'information médiatique dans les pratiques d'enseignement du français, langue étrangère ». Le français dans le monde. Recherches et applications, $\mathrm{n}^{\circ}$ 62. pp. 33-46.

Rakotonoelina, F. (2017b). « Didactique, discours médiatiques et événements : développement d'une compétence pragmatique et d'une culture médiatique par l'analyse de l'actualité ». Les Carnets du Cediscor, $\mathrm{n}^{\circ}$ 13. pp. 33-45. http://journals.openedition.org/cediscor/1012.

Rézeau, J. (2002). « Médiation, médiatisation et instruments d'enseignement : du triangle au “carré pédagogique” ». ASp. pp. 35-36. http://journals.openedition.org/asp/1656.

Shairi, H. R. (2008). « La base éthique de la didactique ». Études de linguistique appliquée, $\mathrm{n}^{\circ} 152$. pp. 485-492. www.cairn.info/revue-ela-2008-4-page-485.htm.

Slakta, D. (1975). «L'ordre du texte ». Études de linguistique appliquée, nº 19. pp. 30-42.

\section{NOTES}

1. Derrière ce recours se pose la question de la place du document authentique en classe de langue. Cette question, d'ailleurs antérieure aux approches communicatives (voir par exemple le numéro datant de juin 1970 du français dans le monde, $\mathrm{n}^{\circ}$ 73), est directement liée aux besoins langagiers des apprenants d'une part - les confronter à des textes produits dans un but communicatif et non pour l'enseignement des langues - pour les placer face à une " communication appropriée aux situations les plus quotidiennes » (Le Goffic et Besse, 1970 : 15) d'autre part.

2. Le Cadre européen commun de référence pour les langues (CECRL), dans une section consacrée au rôle des textes dans l'enseignement et l'apprentissage des langues (2001: 112-113), questionne davantage le recours aux «documents authentiques non trafiqués» (id. : 112) et aux textes "conçus comme matériel pour l'enseignement des langues » (id.) plus qu'elle n'apporte de véritables réponses. Plus précisément, la réflexion sur l'authenticité se déplace des documents/textes vers la notion de tâche, puisqu'il y est désormais question de "tâches authentiques" (l'objectif étant de proposer "plus de tâches et de textes authentiques", id. : 111).

3. À partir d'une étude des textes de presse dans les manuels relevant des approches communicatives (dans une perspective actionnelle ou non), nous avons pu montrer que le choix des thématiques des éditeurs invitait quasi exclusivement à traiter l'aspect culturel du contenu de ces textes (Rakotonoelina, 2017a). Ainsi, parmi les thématiques privilégiées, on notera le repas des Français et la gastronomie, l'immigration, leurs activités culturelles, etc. Or, nous faisions 
remarquer qu'il n'était nul besoin de recourir nécessairement à des textes de presse pour aborder ces réalités culturelles, d'autres textes (hors presse) pouvant également servir cet objectif.

4. L'hypothèse sous-jacente est donc la suivante : l'actualité ne peut faire l'objet d'un traitement en classe de langue que si la thématique de cette actualité considérée au moment de son énonciation peut se répéter à d'autres moments d'énonciation.

5. Dans le CECRL, les trois compétences communicatives langagières sont les suivantes: les compétences linguistiques, la compétence sociolinguistique et la compétence pragmatique. La question des textes à proprement parler est abordée à travers la compétence pragmatique. Ainsi, pour traiter l'actualité, c'est-à-dire la manière dont l'actualité est mise en texte, on s'appuiera sur ce que recouvre cette dernière. De fait, la dimension culturelle dont nous avons précédemment parlé (voir note 3) est davantage associée à la compétence sociolinguistique.

6. Un travail sur la représentation d'une même actualité dans deux ou plusieurs langues-cultures conduit à une démarche transculturelle laquelle permet, comme le souligne Shairi, de «favoriser l'évolution identitaire des sujets apprenants » (2008: 485).

7. Respecter ces préconisations permet à l'enseignant comme à l'apprenant de manipuler des concepts opératoires avec lesquels l'un comme l'autre ont déjà été familiarisés à d'autres occasions, dès lors que la perspective d'enseignement/apprentissage retenue est actionnelle.

8. Cette non-correspondance implique qu'une même actualité (par exemple, un attentat terroriste) n'est pas traitée de la même manière selon les langues-cultures. On se référera par exemple aux travaux de von Münchow (2004) qui montre, dans une perspective comparative, la manière dont l'actualité est traitée dans les journaux télévisés en France et en Allemagne.

9. On peut reprendre ici la distinction entre ce que Benveniste (1974:21) appelle le mode "sémiotique ", le fait qu'il y a d'un côté des formes linguistiques qui doivent être reconnues par ceux qui participent à la communication, et le mode «sémantique ", qui implique de l'autre que l'enchaînement de ces formes doit être interprété en fonction des circonstances.

10. Ce qui n'est plus le cas des textes de la presse quotidienne reprographiés dans les manuels dont on pourrait dire qu'ils seraient a-temporels, conduisant à d'autres formes d'interprétation non liées à cette actualité.

11. Le CECRL distingue la compréhension orale, la lecture ou compréhension écrite et la réception audiovisuelle. Pour la compréhension générale de l'écrit, les descripteurs stipulent : «[p]eut lire avec un grand degré d'autonomie en adaptant le mode et la rapidité de lecture à differents textes et objectifs [...]. Possède un vocabulaire de lecture large et actif mais pourra avoir des difficultés avec des expressions peu fréquentes » (2001: 57). Par ailleurs, s'agissant spécifiquement d'une lecture " pour s'informer et discuter ", les descripteurs précisent : "[p]eut comprendre des articles et des rapports sur des problemes contemporains et dans lesquels les auteurs adoptent une position ou un point de vue particuliers » (2001 : 58). Autrement dit, un travail sur l'actualité présuppose un niveau « avancé ou indépendant » ainsi que le qualifie le Cadre.

12. C'est nous qui soulignons.

13. Le type descriptif est mentionné à la page $98 \mathrm{du}$ CECRL.

14. Alors que l'analyse des actes est une analyse interprétative de reconstruction du sens, l'analyse de reconstruction de la cohérence est une analyse purement linguistique qui montre en quoi le texte d'actualité est un ensemble cohérent. Cette analyse se base elle-même sur les concepts de thème et de progression thématique pour appréhender l'organisation thématique (Slakta, 1975 ; Péry-Woodley, 1993) et les concepts de cohésion grammaticale et lexicale (Halliday et Hasan, 1976 ; McCarthy, 1991 ; Péry-Woodley, 1993).

15. On notera ici que l'enseignant n'est pas là pour porter un jugement de fond sur les articles de presse ni pour participer à un procès éthique ou idéologique de la presse; il doit permettre à l'apprenant d'observer et de comprendre, de façon la plus neutre qui soit, l'actualité de la langue cible. 
16. On rappelle qu'une étude de la cohérence impliquerait également une analyse de l'organisation thématique et de la cohésion grammaticale (voir Rakotonoelina, 2017b).

\section{RÉSUMÉS}

Cet article montre comment développer chez les apprenants une compétence pragmatique permettant une compréhension de l'actualité à partir des textes de la presse écrite quotidienne. Pour cela, on est conduit à distinguer deux types de médiation: une médiation communicationnelle et une médiation didactique. La première permet de comprendre comment l'actualité est mise en scène d'un point de vue discursif et comment il est possible d'en comprendre le sens et de l'interpréter. La seconde met en œuvre, par le biais de l'enseignant, les instruments qui permettent d'analyser cette mise en scène et, pour l'apprenant, de se l'approprier afin d'acquérir une compétence de compréhension de l'actualité. Ainsi, on propose d'aborder celle-ci par le biais de l'événement qu'elle représente et l'on parle alors d'événement médiatique. L'événement médiatique représente à son tour un type d'événement et l'objectif est alors de décrire ce type de deux points de vue: du point de vue de sa visée illocutoire (compétence fonctionnelle) et du point de vue des formes langagières considérées comme des indices relevant de la cohérence (compétence discursive). Il en découle des schématisations discursives propres à chaque type d'événement permettant aux apprenants d'aborder l'actualité de la langue cible avec plus d'aisance.

This paper deals with a methodological approach to develop the learner's pragmatic competences in media information texts and discourse in French as a foreign language. Two types of mediation can be considered: a communicational mediation and a didactic mediation. The purpose of the first one is to explain how news is turned into discourse, and how to understand and interpret their meaning. The second one, in which the teacher takes center stage, brings the necessary tools to analyze media information texts and discourse to help learners understand and interpret them. News is based on real events, but the analysis reveals how those events function as media events. Media events can be typified and be described in terms of illocutionary acts (corresponding to the functional competence) and in terms of coherence and cohesion (corresponding to the discourse competence). The analysis leads to discursive schematizations for every media event, which facilitate the learner's understanding of media information.

\section{INDEX}

Mots-clés : didactique du FLE, médiation communicationnelle, médiation didactique, discours d'information médiatique, compétence pragmatique

Keywords : Language learning and teaching, discourse analysis, pragmatic competence, media event, understanding, news, newspapers 


\section{AUTEUR}

\section{FLORIMOND RAKOTONOELINA}

Université Paris 3 - Sorbonne nouvelle

EA 7345 CLESTHIA - CEDISCOR

Florimond Rakotonoelina est maître de conférences en sciences du langage et enseigne au département de didactique du français, langue étrangère et seconde, à l'université Paris 3 Sorbonne nouvelle. Il est rattaché à l'EA 7345 - CLESTHIA Langage, systèmes, discours. Il dirige la publication non périodique les Carnets du Cediscor (Presses Sorbonne Nouvelle et OpenEdition). Ses recherches s'ancrent dans le courant de l'analyse du discours et portent actuellement sur les discours médiatiques, les discours didactiques et les discours de transmission des connaissances en général, notamment en e-learning. 